\title{
Stability as an Overhaul: Inspecting Cloud Stability
}

\author{
M.Nanda Kishore \\ MCA, M Tech, \\ Assistant Professor, \\ Department of MCA, \\ Sri Venkateswara College of \\ Engineering and Technology, \\ Chittoor, India
}

\author{
U. Lakshmi Priya \\ PG Scholar, \\ Department of MCA, \\ Sri Venkateswara College of \\ Engineering and Technology \\ Chittoor, India
}

\author{
T.M.Theja Sree \\ PG Scholar, \\ Department of MCA, \\ Sri Venkateswara College of \\ Engineering and Technology, \\ Chittoor, India
}

\begin{abstract}
Since the distributed storage administrations are getting more renowned as a result of their preferences, so as to give worldwide access to the information which is display on the cloud, a supplier called "Cloud Service Provider (CSP)" keeps up various imitations for every bit of information on distributed servers. The significant issue of utilizing this replication system as a part of mists is that it is extremely lavish to accomplish solid consistency. Subsequently, to conquer this circumstance we first present a model consistency as a Service (CaaS), which comprises of an extensive information cloud and various review mists. In this model, an information cloud is kept up by a CSP, and a gathering of clients who makes up a review cloud can check whether the information cloud gives the expected level of consistency or not. Additionally we have proposed a two-level examining building design, which just needs an inexactly synchronized check in the review cloud. At last, we define a Heuristic Auditing Strategy (HAS) to uncover however many infringement as could be expected under the circumstances. HAS can be approved by genuine cloud organizations.
\end{abstract}

\section{General Terms}

Stability as an overhaul, synchronized check, inspecting cloud stability, worldwide inspecting.

\section{Keywords}

Consistency as a Service (CaaS), two-level Auditing Structure, heuristic Auditing Strategy (HAS) and Cloud Storage.

\section{INTRODUCTION}

Distributed computing has ended up financially better known, as it pledges to ensure versatility, flexibility, and high accessibility and at an expense which is low. Started by the pattern of the everything-as-a Service model. Information stockpiles, virtualized framework, virtualized stages, and also applications and virtual products are being given and devoured as administrations in the cloud. Distributed storage administrations can be incorporated as an unmistakable administration in distributed computing, which considers the conveying the information stockpiling as a Service. All the more frequently charged on an utility registering premise.. A portion of the cases might incorporate Amazon SimpleDB, Microsoft Azure stockpiling, and so on. By utilizing the distributed storage benefits, the information put away in the cloud can be gotten to whenever and anyplace, utilizing any gadget, without much thinking about a lot of speculations when sending the fundamental equipment.
Distributed computing has ended up economically popular, as it guarantees to ensure versatility, flexibility, Guided by the pattern of the everything-as-an administration (XaaS) model, information stockpiles, virtualized framework, virtualized stages, also as software and applications are being given and expended as services in the cloud. Distributed storage administrations can be regarded as a run of the mill benefit in distributed computing, which includes the delivery of information stockpiling as an administration, including database-like services and system appended capacity, regularly charged on an utility processing premise, e.g., every gigabyte every month. Examples include Amazon SimpleDB1, Microsoft Azure storage2, and so on. By utilizing the distributed storage benefits, the customers can access information put away in a cloud at whatever time and anyplace. Utilizing any gadget, without thinking about a huge sum of capital investment when sending the hidden hardware infrastructures. To meet the guarantee of universal $24 / 7$ get to, the cloud administration supplier (CSP) stores information reproductions on numerous geologically conveyed servers. A key issue of utilizing the replication method as a part of mists is that it is exceptionally expensive to accomplish solid consistency on an overall scale, where an user is guaranteed to see the most recent upgrades. Really, mandated by the CAP principle3, numerous CSPs (e.g., Amazon S3) just guarantee powerless consistency, for example, possible consistency, for performance and high accessibility, where a client can read stale information for a time of time. The area name framework (DNS) is a standout amongst the most mainstream applications that implement eventual consistency. Redesigns to a name won't be visible immediately, yet all customers are guaranteed to see them eventually. However, consequent consistency is not a catholicon for all applications. Especially for the intelligent applications, stronger consistency affirmation is of expanding significance. Cloud gives just consequent consistency, then Bob is permitted to get to an old rendition of the necessity investigation from CS5.In this case, the coordinated configuration that is in view of an old adaptation may not fulfill the genuine necessities of clients. Really, diverse applications have distinctive consistency necessities. For instance, mail administrations need monotonic read consistency and read-your-compose consistency, however interpersonal organization administrations need causal consistency. In distributed storage, consistency decides accuracy as well as the genuine expense every exchange. In this paper, we show a novel consistency as an administration (CaaS) model for this circumstance. The CaaS model comprises of an expansive information cloud and various little review mists. The information cloud is kept up by a CSP, and a review cloud comprises of a gathering of clients that chip in on an occupation, e.g., a report or an undertaking. An administration level understanding (SLA) will be locked in 
the middle of the information cloud and the review cloud, which will stipulate what level of consistency the information cloud ought to give, and how much (financial or generally) will be charged if the information cloud disregards the SLA. The usage of the information cloud is dark to all the clients because of the virtualization strategy. In this way, it is hard for the clients to confirm whether every imitation in the information cloud is the most recent one or not. Roused by the arrangement in, we permit the clients in the review cloud to check cloud consistency by examining a hint of intelligent operations. Not at all like their work, we don't oblige a worldwide clock among all clients for aggregate requesting of operations. we propose a heuristic Auditing Strategy (HAS) which adds proper peruses to uncover however many infringement as would be prudent. Our key commitments are as per the following:

We exhibit a novel consistency as a Service (CaaS) model, where a gathering of clients that constitute a review cloud can check whether the information cloud gives the guaranteed level of consistency or not. We propose a two-level evaluating structure, which just obliges an approximately synchronized check for requesting operations in a review cloud.

We plan calculations to measure the seriousness of infringement with distinctive measurements. We devise a heuristic Auditing Strategy (HAS) to uncover however many infringement as could be expected under the circumstances. Broad examinations were performed utilizing a mix of reproductions and a genuine cloud arrangement to approve HAS.

\section{EXISTING SYSTEM}

In the current framework, to meet the guaranteed level of worldwide get to all the time, a cloud administration supplier (CSP) stores information replications on different servers. A real issue of utilizing the replication method as a part of mists is that it is extremely extravagant to accomplish solid consistency around the world, where a client is guaranteed to see the most recent upgrades. Really, numerous CSPs (e.g., Amazon S3) just guarantee powerless consistency, for instance possible consistency, for execution and high accessibility, where a client can read the information which is not unique for a time of time. The space name framework (DNS) is one of the mainstream applications that actualize consequent consistency. Overhauls that are made to a name won't be obvious promptly, yet all the customers are guaranteed to see them inevitably. Particularly more elevated amount significance is given for the intuitive applications. Assume that Alice and Bob are dealing with an application utilizing a distributed storage administration, where the majority of the related data is imitated to five cloud servers, CS1,...., CS5. In the wake of transferring another variant of the application to a CS4, Alice calls Bob to download the most recent rendition. Here, after Alice calls Bob, the causal relationship is created between Alice's redesign and Bob's perused. Accordingly, the cloud must give causal consistency, which guarantees that Alice's overhaul is focused on the majority of the copies before Bob's perused. In the event that the cloud gives just inevitable consistency, then Bob is permitted to get to a more seasoned variant of the application from CS5. In this circumstance, the application that is in view of an old variant may not fulfill the genuine prerequisites of clients.
Actually, distinctive applications have diverse consistency prerequisites. In distributed storage, consistency decides accuracy as well as the real cost every exchange which is being made. In this paper, we propose consistency as a Service (CaaS) model for this circumstance.

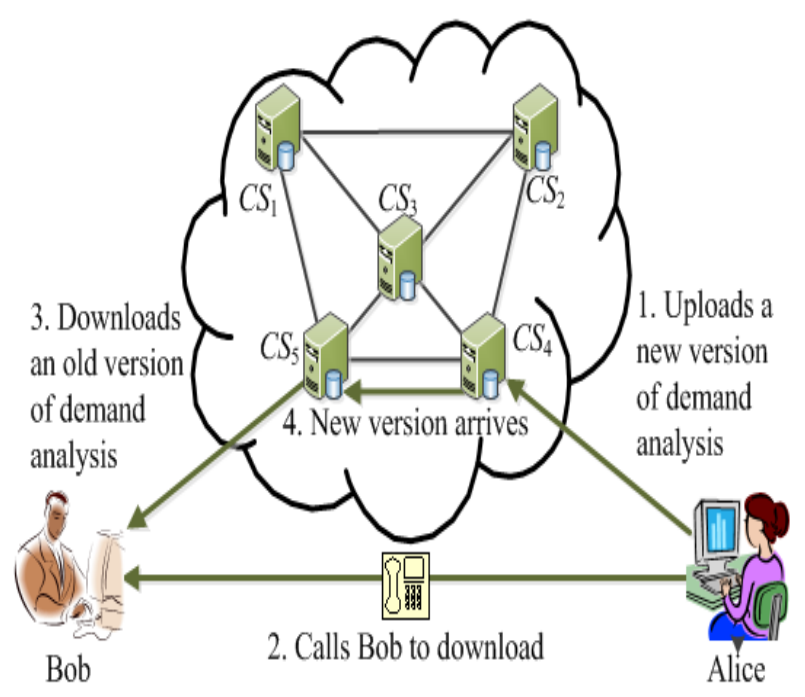

Figure 1: An application that requires causal consistency

\section{PROPOSED SYSTEM}

The CaaS model comprises of a vast information cloud and various little review mists. The information cloud is kept up by a CSP, and a review cloud comprises of a gathering of clients that coordinate on an occupation, e.g., a report or a venture.

A Service level assertion (SLA) will be locked in the middle of the information cloud and the review cloud, which will show what level of consistency the information cloud ought to give, and the amount of will be charged if the information cloud disregards the SLA.

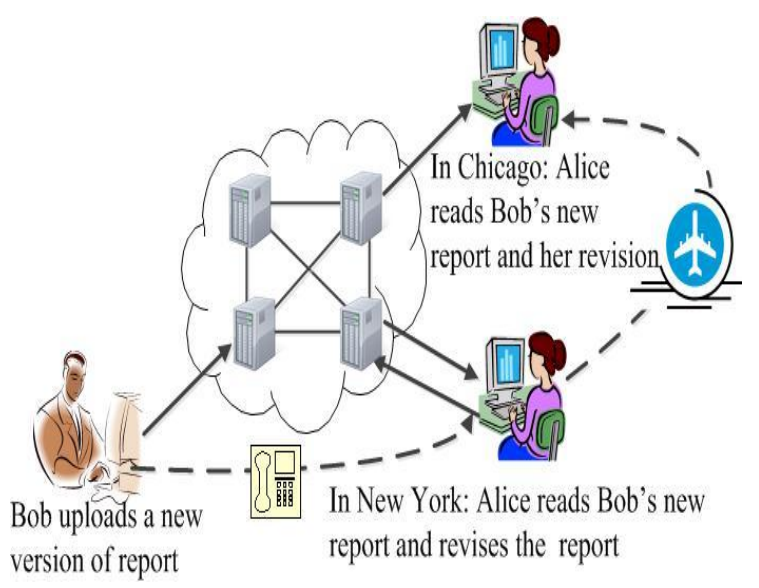

Figure 2: An application that has different consistency
requirements

The execution of the information cloud is strong to all the clients. Along these lines, it is hard for the clients to check whether every reproduction in the information cloud is the most recent one or not. Dissimilar to, and we receive a twolevel examining structure: every client can perform nearby reviewing freely with a neighborhood hint of operations; 
intermittently, an evaluator is chosen from the review cloud to perform worldwide evaluating. We propose a heuristic evaluating procedure (HAS) which uncovers whatever number infringement as could reasonably be expected. Our key commitments are as per the following:

$>\quad$ We present a novel consistency as a Service (CaaS) model, where a gathering of clients that constitute a review cloud can confirm whether the information cloud gives the guaranteed level of consistency or not.

We propose a two-level auditing structure, which just obliges an approximately synchronized clock for requesting operation erations in a review cloud.

$>$ We outline calculations to evaluate the seriousness of infringement.

$>\quad$ We devise a Heuristic Auditing Strategy (HAS) to uncover whatever number infringement as could reasonably be expected.

The CaaS model comprises of a cloud also, different review mists. The information cloud, kept up by the cloud Service Provider (CSP), is a key-esteem information stockpiling framework, where every bit of information is distinguished by a special key. To give dependably on administrations, the CSP reproduces the majority of the information on various topographically conveyed cloud servers.

A review cloud comprises of a gathering of clients that coordinate on a vocation, e.g., a report or a system. We accept that every client in the review cloud is distinguished by a special ID. Before outsourcing the employment to the information cloud, the review cloud and the information cloud will hold in a Service level Agreement (SLA), which stipulates the guaranteed level of consistency that ought to be given by the information cloud. The review cloud exists to check whether the information cloud abuses the SLA or not, and to evaluate the seriousness of infringement.

\section{SYSTEM ARCHITECTURE}

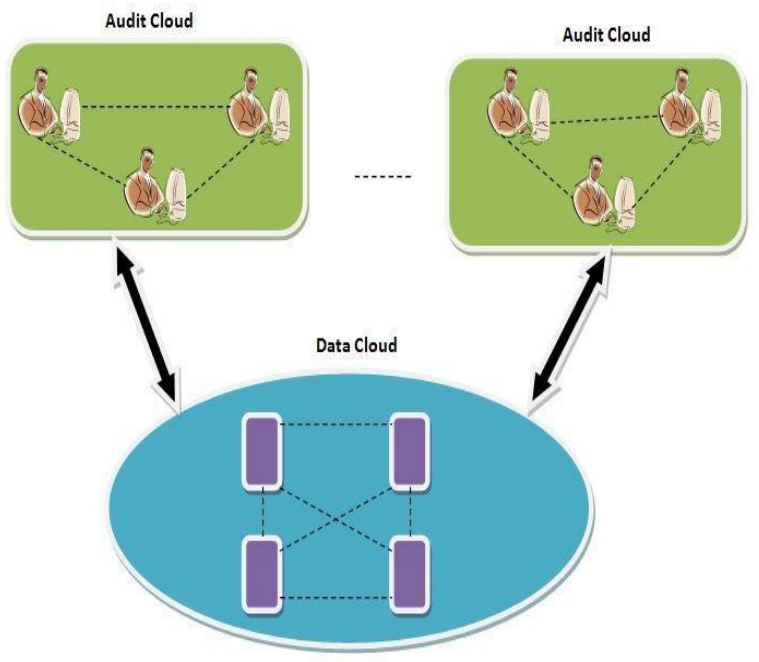

Figure 3: System Architecture Diagram
At the first level, every client performs nearby examining. Different applications will have diverse consistency necessities. At the second level, an examiner can perform worldwide inspecting in the wake of getting a worldwide hint of every one of clients' operations. In the application stage, in the wake of transferring another variant of the report to the information cloud, Bob calls Alice, requesting that her download it. After the call, Bob's upgrade and Alice's perused are causally related. Thusly, causal consistency obliges that Alice must read Bob's new report. The principle preferences of this proposed framework is that:

$>$ The clients can ready to survey the nature of cloud administrations.

$>$ Able to pick the privilege CSP among various applicants, e.g., the slightest costly one that still gives sufficient consistency to the users applications.

\section{HEURISTIC AUDITING STRATEGY}

A heuristic procedure is a development to a critical thinking, learning, or revelation that utilizes a down to earth strategy which is not ensured to be ideal or flawless, but rather sufficient for the prompt objectives. Heuristic routines can be utilized to accelerate the methodology of discovering an acceptable arrangement. Hence, the fundamental thought of our heuristic examining methodology (HAS) is uncovering however many infringement as could be allowed.
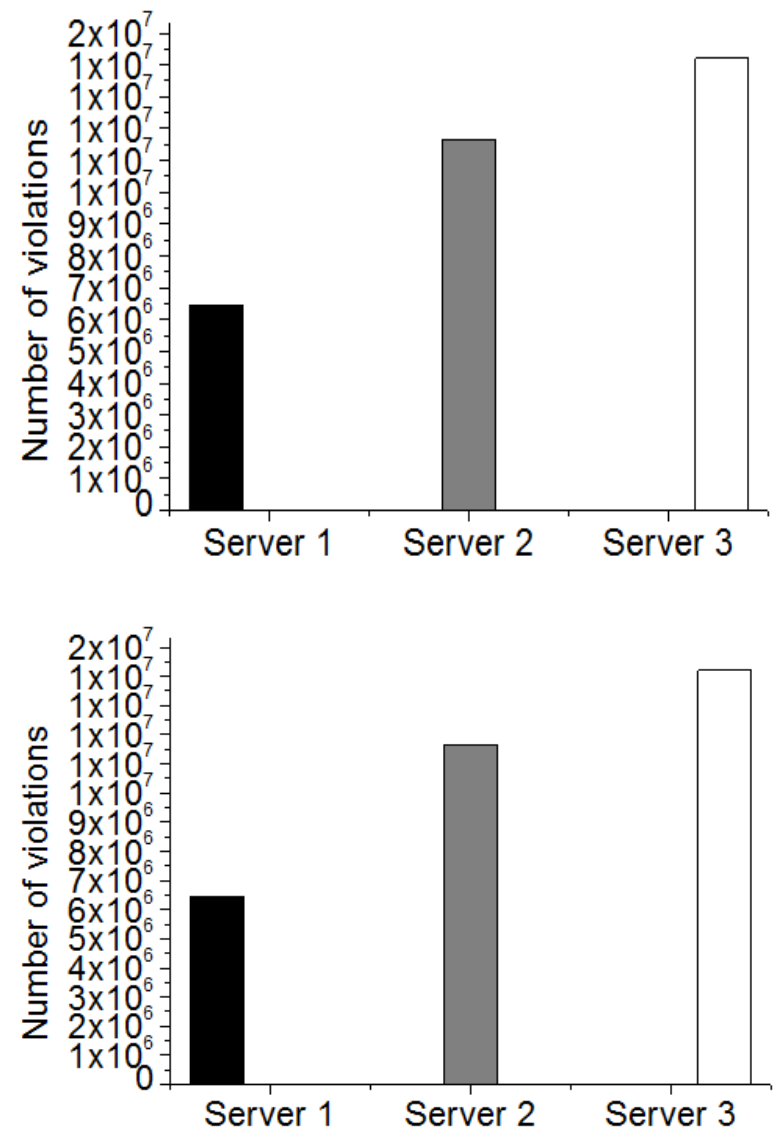

Figure 4: Average delays and violations in Cloud 


\section{RESULTS AND DISCUSSIONS}

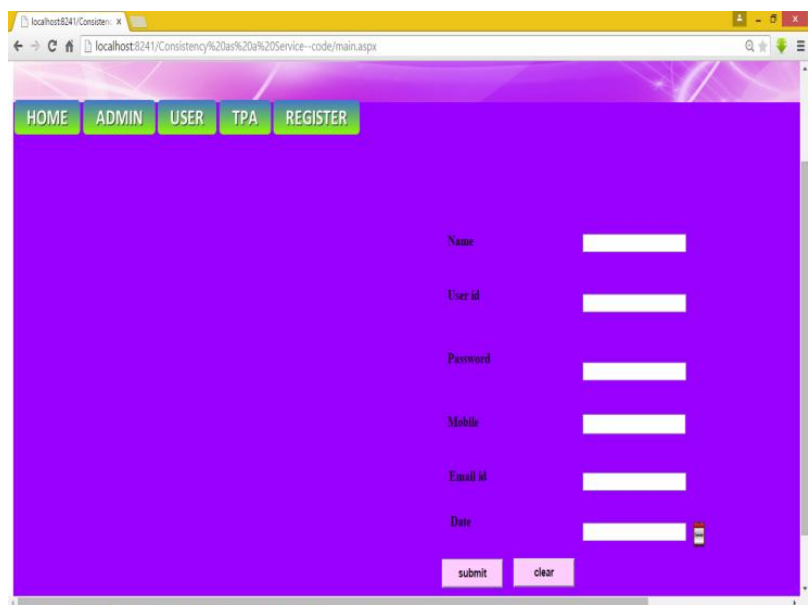

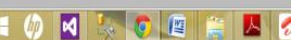

Figure 6.1: Registration Page

This Registration Page is used to register the details of user. Once the user has registered, he can login to the cloud for performing further actions.

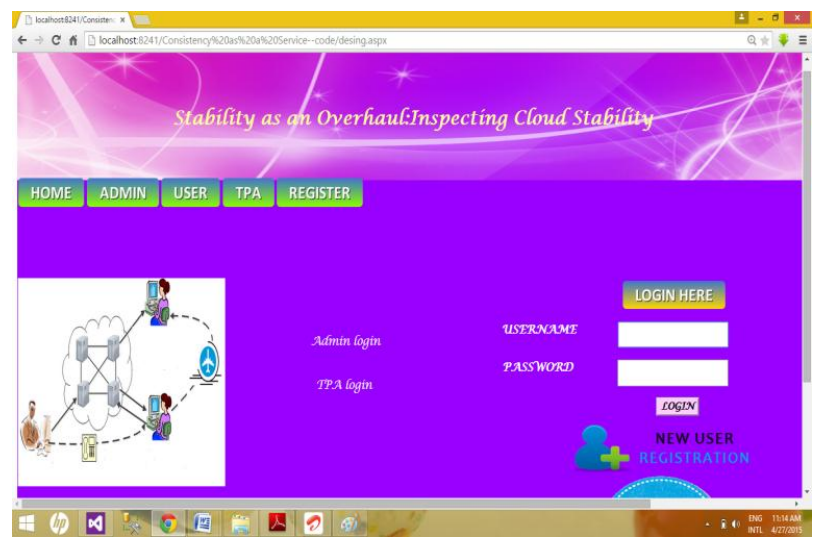

Figure 6.2: Login Page

Users who have registers can login using his credentials and once he has logged in, he can upload a file or even he can view the files that are uploaded.

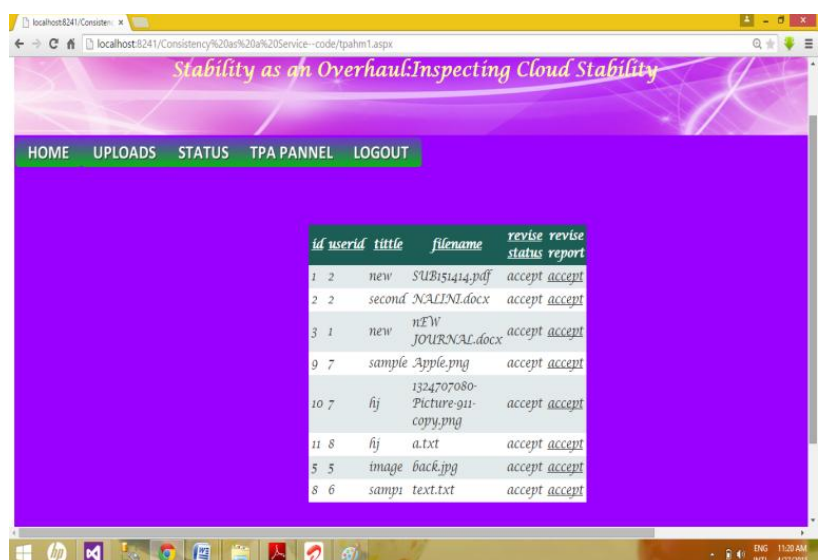

Figure 6.3: Auditors Page
The auditor is a person in the audit cloud who can perform auditing on the files which are being uploaded by the user.

\section{CONCLUSION}

In this paper, we displayed a consistency as a Service (CaaS) model and a two-level examining structure that help clients confirm whether the cloud administration supplier (CSP) is giving the guaranteed consistency or not, and to quantify the seriousness of the infringement, if any. With the CaaS model, the clients can survey the nature of cloud administrations and pick a privilege CSP among different choices, for e.g., the slightest costly onto that still gives sufficient consistency to the client's applications.

\section{ACKNOWLEDGEMENTS}

We thank our project guide Mr.M.Nanda kishore for their valuable guidance and for providing all the necessary facilities, which were indispensable in completion of this paper for helpful discussions and comments.

\section{REFERENCES}

[1] M. Armbrust, A. Fox, R. Griffith, A. Joseph, R. Katz, A. Konwinski,G. Lee, D. Patterson, A. Rabkin, I. Stoica, et al., "A view of cloud computing," Commun. ACM, vol 53, no. 4, 2010.

[2] P. Mell and T. Grance, "The NIST definition of cloud computing (draft),"NIST Special Publication 800-145 (Draft), 2011.

[3] E. Brewer, "Towards robust distributed systems," in Proc. 2000 ACMPODC.

[4] "Pushing the CAP: strategies for consistency and availability," Computer, vol. 45, no. 2, 2012.

[5] M. Ahamad, G. Neiger, J. Burns, P. Kohli, and P. Hutto, "Causalmemory: definitions, implementation, and programming," DistributedComputing, vol. 9, no. 1, 1995.

[6] D. Bermbach and S. Tai, "Eventual consistency: how soon is eventual?" in Proc. 2011 MW4SOC. 